Model identification for impact dynamics of a piezoelectric microactuator

This article has been downloaded from IOPscience. Please scroll down to see the full text article.

2012 J. Micromech. Microeng. 22115002

(http://iopscience.iop.org/0960-1317/22/11/115002)

View the table of contents for this issue, or go to the journal homepage for more

Download details:

IP Address: 141.211.173.82

The article was downloaded on 25/06/2013 at 20:33

Please note that terms and conditions apply. 


\title{
Model identification for impact dynamics of a piezoelectric microactuator
}

\author{
Jeong Hoon Ryou and Kenn Richard Oldham \\ Department of Mechanical Engineering, University of Michigan, 2350 Hayward, Ann Arbor, \\ MI 48109, USA \\ E-mail: jhryou@umich.edu and oldham@umich.edu
}

Received 5 April 2012, in final form 17 August 2012

Published 18 September 2012

Online at stacks.iop.org/JMM/22/115002

\begin{abstract}
A parameterized model for the impact dynamics of a piezoelectric microactuator is proposed, and a system-identification procedure for quantifying model parameters is presented. The proposed model incorporates squeeze-film damping, adhesion and coefficient-of-restitution effects. Following parameter quantification from sample data of bouncing impacts and progressive ramped-square-wave inputs, the model is found to be effective in predicting the time response of the actuator to a range of square-wave and sinusoidal inputs. The main contributions of this paper are to show that the dynamic response to micro-scale contact can be predicted using simple lumped-parameter modeling after a proposed system-identification procedure is performed and that certain small-scale forces can be quantified. For example, for motions where bounce of the cantilever tip may occur, the range of adhesion is found to be time dependent and vary between approximately 20 and $520 \mathrm{nN}$, while the range of squeeze-film damping is estimated to be between 50 and $130 \mathrm{nN}$, depending on the input signal frequency and amplitude. The presence, absence and quantity of bounces upon impact are predicted very accurately, while oscillation amplitudes and contact durations are predicted to be between $1 \%$ and $30 \%$ error for the majority of many test cases of periodic inputs between 5 and $100 \mathrm{~Hz}$.
\end{abstract}

\section{Introduction}

Contact dynamics of microsystems are often complex and difficult to model, due to the existence of various smallscale, nonlinear forces affecting the behavior of micro-scale objects before, during and after contact. While various models have been proposed for different physical situations, when a microstructure must interact with its external environment, it may be especially useful to obtain a dynamic model without full knowledge of the geometry or properties of the interacting surfaces. In this paper, a model is developed for contact dynamics of a silicon proof mass driven by a piezoelectric actuator into contact with an underlying, irregular silicon 'ground' surface. The geometry of the proof mass and the piezoelectric forcing are intended to approximate the contact between the foot of a terrestrial micro-robot and the terrain on which it operates. Methods for modeling and/or identifying the forces acting on such a robot foot are potentially very useful for predicting walking gait performance of prototype micro-robots based on piezoelectric thin films.
Previous studies of contact behavior of MEMS devices have most often relied on accurate knowledge of interacting surface geometries. This is especially true for studies of scanning probe technologies, as in atomic force microscopy [1] or probe storage research [2], which has allowed extremely detailed studies of contact dynamics for such instruments. For applications with larger interacting surfaces, on the order of $10-1000 \mu \mathrm{m}$, the most prevalent area of contact modeling is for micro-electromechanical switches [3, 6-13], with additional work being done on certain vibration scavenging [4] or miniature gear devices [5]. Table 1 shows a summary of many contact models from the literature for interacting surfaces at this scale. In all cases, the geometries of the interacting surfaces are taken to be well known, and nearly all cases involve electrostatic forcing. A variety of models for impact, adhesion and damping behavior have been utilized, and these have enabled accurate predictions of certain specific phenomena during one or more impact events. More specifically, among closely related models in table 1, Decuzzi [7] and Do [8] investigated trends in adhesion/contact force for different environments and Do [8], LaRose [10] and McCarthy [11] 
Table 1. Comparison of features included in contact models for impact of fixed surface and actuated device $0.01-1 \mathrm{~mm}$ in size. Abbreviations: ES—electrostatic; EX—external; SF—squeeze film; PE—piezoelectric; L—lumped dynamics; D—distributed dynamics; $\mathrm{M}$-modal dynamics; CoR—coefficient of restitution; VdW-Van der Waals; TD—time dependent (empirical).

\begin{tabular}{lllllll}
\hline Authors & Forcing & Dynamics & Damping & Impact & Adhesion & Experimentally validated \\
\hline Wang et al $[$ [6] & ES & L & SF & Asperity & - & Threshold for bouncing \\
Decuzzi et al [7] & ES & D & SF & VdW & VdW & - \\
Do et al [8] & ES & D & SF & Elastic/plastic & - & Periods of contact \\
Park et al [9] & ES & M & SF & Lagrange & - & Feedforward control gains \\
LaRose et al [10] & ES & D & SF & CoR & - & Transient response match \\
McCarthy et al [11] & ES & D & SF & Spring & - & Bounce times versus voltage \\
Granaldi et al [12] & ES & L & L & VdW & VdW & - \\
Zhang et al [13] & ES & L & L & Spring & - & Freq. response amplitude \\
Guo et al [3] & ES & D & SF & VdW & VdW & Open/closed switch times \\
Czapleski et al [14] & ES & L & L & - & - & Transient before impact \\
Field \& Epp [4] & EX & M & L & Stochastic & - & Statistical properties \\
Current work & PE & L & SF & CoR & TD & Time response trajectory for a \\
& & & & & & range of periodic inputs. \\
\hline
\end{tabular}

suggested novel impact/bounce models over various substrate conditions, which are used to inform the lumped-parameter modeling in this paper. Additionally, for models based on an assumption that the geometry of the contact surfaces is well known, close estimation of the short-ranged forces was conducted in several studies, including use of the Reynolds equation for squeeze-film damping $[3,6,7,10,11]$ and various adhesion/contact force models such as Johnson-KendallRoberts (JKR) [3] or Lennard-Jones force [12]. However, a common drawback in these models, besides the a priori knowledge of geometry, is the absence of explicit experimental validation of the model over prolonged periods in the time domain, which is crucial for the analysis of the influence of various small-scale nonlinear forces during locomotion of a terrestrial micro-robot.

The approach taken in the following study is to attempt to model contact behavior using simplified and parameterized models for relevant interaction forces and to apply systemidentification techniques to quantify these parameters without detailed knowledge of the interacting surface geometries or properties. This approach allows a wider variety of impact phenomena to be predicted than has been achieved by most other models for devices with similar dimensions; these phenomena include the presence of bouncing events, contact duration and oscillation amplitudes during periodic operation. Naturally, the limitation of this approach relative to prior works is that the model parameters cannot at this time be predicted from basic material properties, although prior works provide information on which small-scale factors to include. A second limitation of the current model is that fundamental underlying forces, such as the Van der Waals force, may be obscured in lumped parameter representations. Meanwhile, another advantage and difference between this model and other prior models is that electrostatic forcing, one of the dominant factors in most micro-electromechanical switch models, is nearly negligible for the piezoelectric test structure being analyzed, which in some situations makes other small-scale phenomena more impactful on device response.

\section{Test structure and instrumentation}

The test structure used for contact model development consists of a simple lead-zirconate-titanate (PZT) unimorph attached to a rigid silicon proof mass, as shown in figure 1. The unimorph is a composite thin-film stack of a silicon dioxide base layer, platinum bottom electrode, chemical-solution-deposited PZT active layer, top platinum electrode and structural gold layer. The active portion of the cantilever is $750 \mu \mathrm{m}$ long and $100 \mu \mathrm{m}$ wide, while the proof mass is $150 \mu \mathrm{m}$ long and $100 \mu \mathrm{m}$ wide, and is formed from the device layer of a silicon-on-insulator wafer with $10 \mu \mathrm{m}$ thickness. The proof mass dimension and first resonant frequency of the test structure are selected to approximately match the foot dimension and first resonant frequency of prototype micro-robotic leg joints [15]. The test structure was produced by the same fabrication process as robot prototypes and shown in figure $1(b)$; the fabrication process is the same as that previously presented in [16, 17] and was performed at the US Army Research Laboratory.

The cantilever test structure is released from the silicon substrate in an isotropic $\mathrm{XeF}_{2}$ silicon etch, which gives rise to an irregular and unknown silicon geometry beneath the proof mass. Identical test structures with the cantilever physically removed after fabrication indicate that the primary contact region is a ridge at the center of the proof mass, as would be expected from isotropic etching, but exact length and height vary from device to device. During operation, the bottom platinum electrode is used as ground to minimize electrostatic forces between cantilever and ground, helping to isolate contact behaviors, while the active voltage input is applied to the top platinum electrode. During experimentation, static displacement of the cantilever was measured using an optical profilometer, (LEXT OLS4000), while dynamic displacements were measured at the center of the cantilever and center of the proof mass using a laser Doppler vibrometer (Polytek PSV400). Figure 2 shows a diagram of the experimental setup.

\section{System model}

The system model consists of a lumped parameter model approximating the modal dynamics of the test structure, 


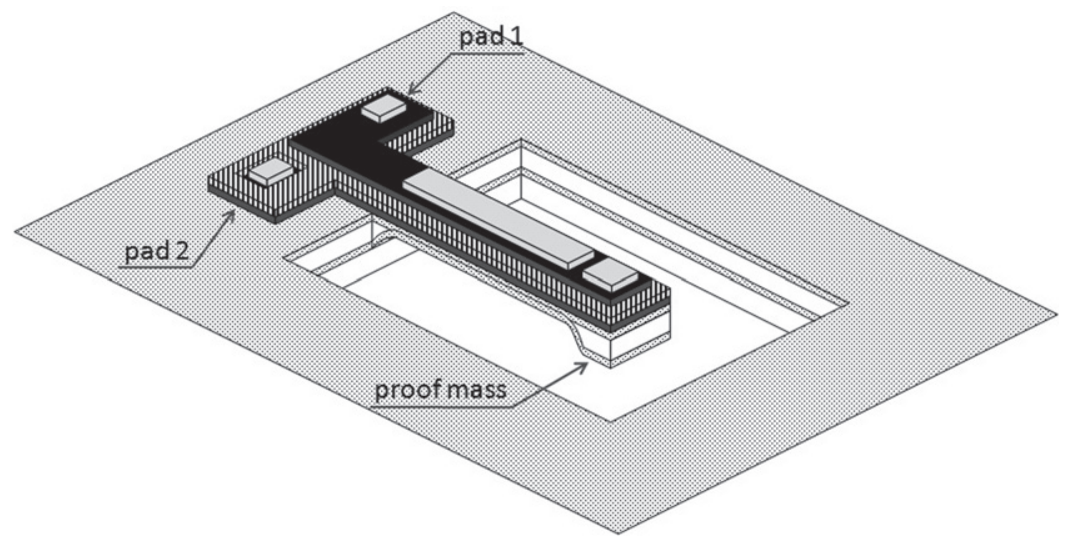

(a)

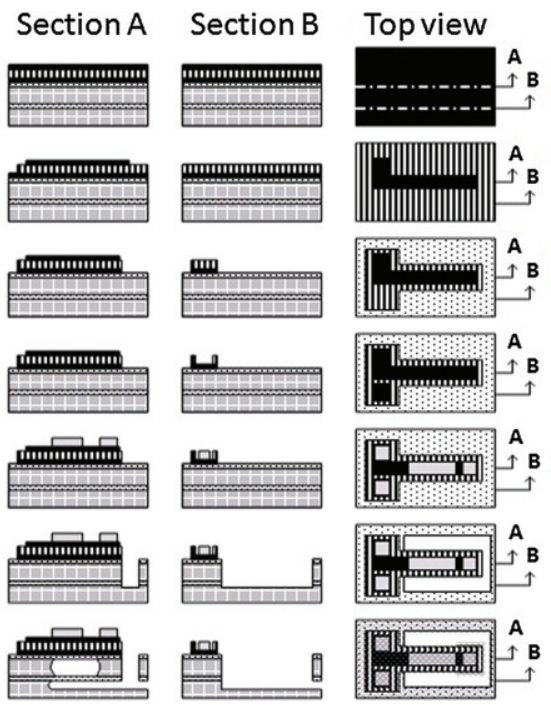

$\boxplus \mathrm{Si} \quad$ 組 PZT $\quad \mathrm{Ti} / \mathrm{Pt}$

$\mathrm{SiO}_{2} \quad \square \mathrm{Au} \quad \square$ Photoresist (shown

only in top view)

(b)

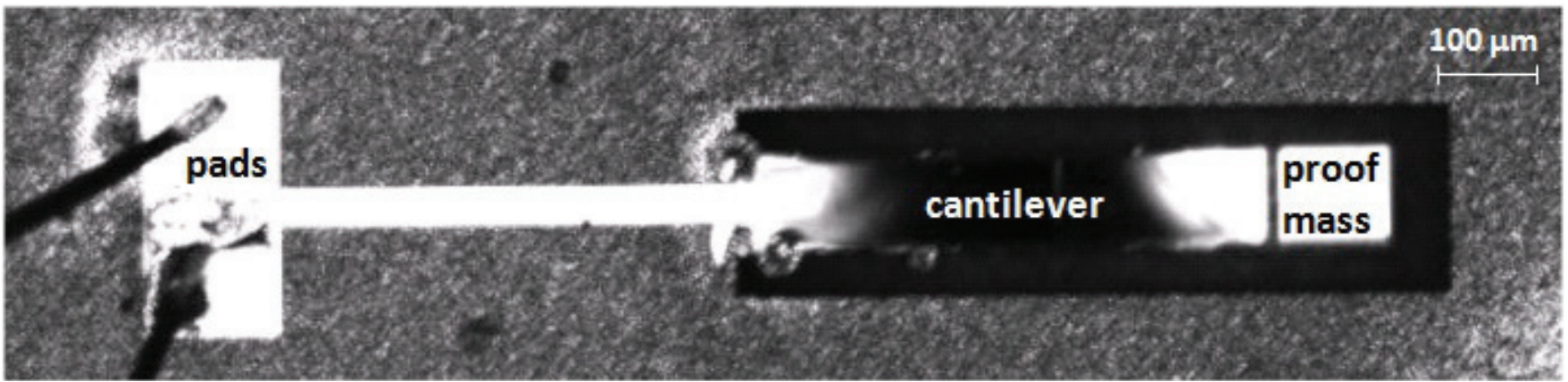

$(c)$

Figure 1. (a) Micro-cantilever test structure, (b) fabrication process and (c) image of cantilever from optical microscope.

an empirically derived model for internal forcing applied by the piezoelectric thin film on the cantilever beam, and parameterized squeeze-film damping, adhesion and impact models for ground interaction.

\subsection{Structural dynamics}

The structural dynamics of the cantilever beam and proof mass are modeled in the form

$$
\mathbf{M} \ddot{\mathbf{x}}+\mathbf{B} \dot{\mathbf{x}}+\mathbf{K} \mathbf{x}=\mathbf{U},
$$




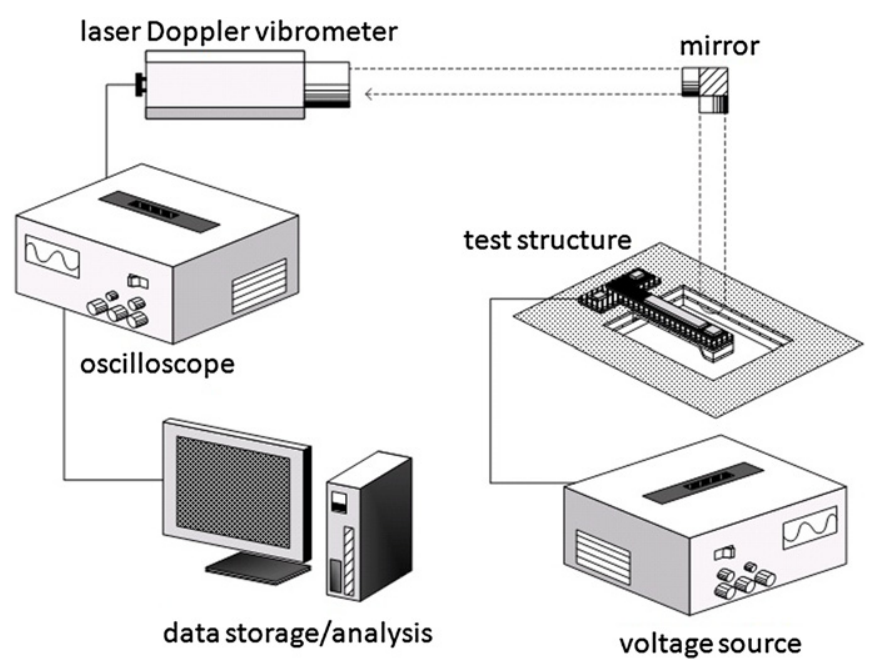

(a)

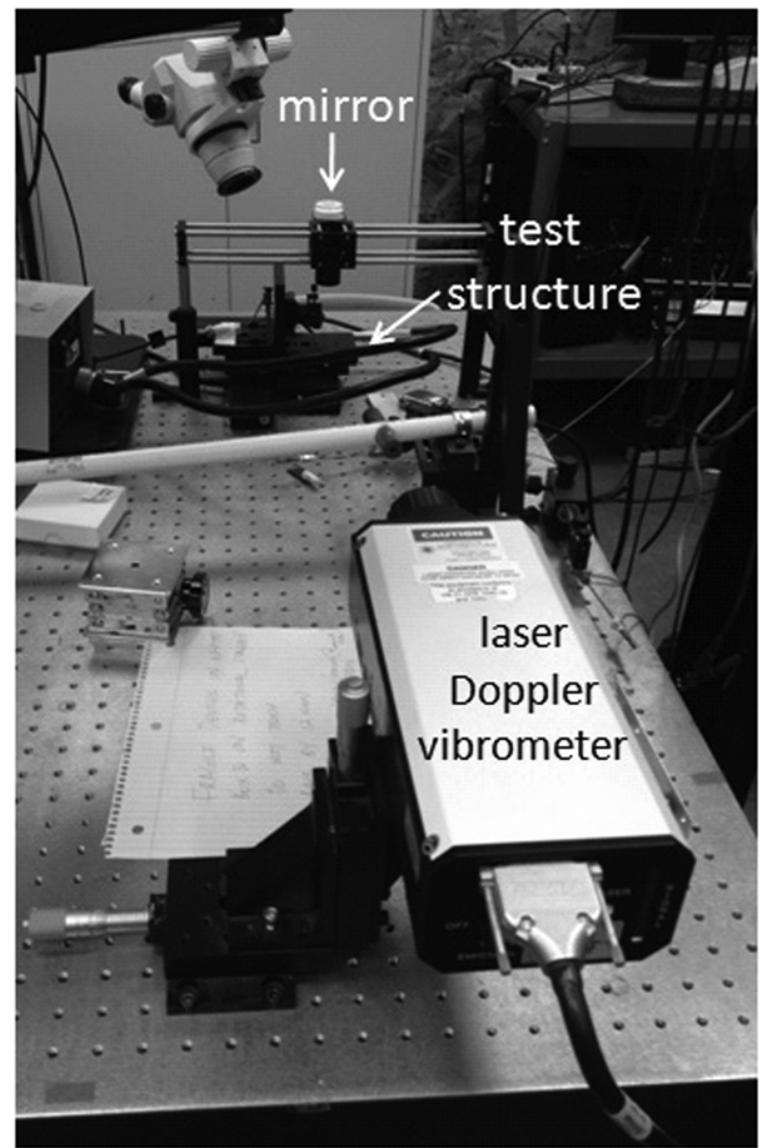

(b)

Figure 2. Measurement instruments and experimental setup: (a) schematic view and $(b)$ photograph of the laser Doppler vibrometer setup.

where $\mathbf{M}, \mathbf{B}$ and $\mathbf{K}$ are linear mass, damping and stiffness matrices, respectively, and $\mathbf{U}$ is a vector of forcing inputs. For convenience, the state vector of the system $\mathbf{x}$ is chosen to be of second order, with states $x_{\mathrm{c}}$, displacement at the center of the cantilever, and $x_{\mathrm{p}}$, displacement at the center of the proof mass (i.e. $\mathbf{x}=\left[\begin{array}{ll}x_{\mathrm{p}} & x_{\mathrm{c}}\end{array}\right]^{\mathrm{T}}$ ). While this allows only the two most prominent vibration modes of the system to be captured in the dynamic model, both these states are simple to measure using available instrumentation, and the forcings to the system are approximately decoupled into forces acting on the cantilever and proof mass, respectively. This allows $\mathbf{U}$ to be modeled in the form

$$
U=\left[\begin{array}{c}
u_{\mathrm{c} 0}+u_{\mathrm{c}, \mathrm{pzt}}\left(V\left(t^{-} \mid t^{-}<t\right)\right) \\
u_{\mathrm{p} 0}+u_{\mathrm{p}, \mathrm{g}}\left(x_{\mathrm{p}}, \dot{x}_{\mathrm{p}}, t\right)
\end{array}\right],
$$

where $u_{\mathrm{c} 0}$ and $u_{\mathrm{p} 0}$ are fixed, constant sources of structural deformation due to residual stresses from the fabrication process, $u_{\mathrm{c}, \mathrm{pzt}}$ is the sum of internal driving forces in the cantilever beam, which is a function of the applied voltage to the system $V$ and influenced by the history of that voltage input up to the current time $t$, and $u_{\mathrm{p}, \mathrm{g}}$ is the sum of external forces generated through interaction with the ground, which depends on position and velocity of the proof mass.

Because the neutral position of the system when no voltage is applied is dependent on the voltage history of the piezoelectric film, the zero positions for $x_{\mathrm{p}}$ and $x_{\mathrm{c}}$ are defined by applying a specified voltage function prior to each identification experiment, namely a polarization signal $V_{\text {pole }}(t)$ represented by

$$
V_{\text {pole }}(t)=\left\{\begin{array}{ll}
10, & t<0 \\
0, & t \geqslant 0
\end{array} .\right.
$$

Then, the neutral position of the system is defined as the solution to

$$
\mathbf{K x}_{0}=\left[\begin{array}{c}
u_{\mathrm{c} 0}+u_{\mathrm{c}, \mathrm{pzt}}\left(V_{\text {pole }}(0)\right) \\
u_{\mathrm{p} 0}
\end{array}\right]
$$

or in other words, zero positions are the position of the cantilever and proof mass in air after poling the PZT film at $10 \mathrm{~V}$ (in practice, $10 \mathrm{~V}$ is applied for $10 \mathrm{~min}$, with at least $1 \mathrm{~min}$ before measurement of cantilever and proof mass position by optical profilometry or LDV). Then, the dynamic system to be analyzed in terms of contact dynamics is described by differential states $\delta \mathbf{x}=\mathbf{x}-\mathbf{x}_{\mathbf{0}}$ and $\delta \mathbf{u}=\mathbf{u}-\mathbf{u}_{\mathbf{0}}$ and differential forces $\delta u_{\mathrm{c}}(t)$ and $\delta u_{\mathrm{p}}(t)$ :

$$
\begin{aligned}
\mathbf{M} \delta \ddot{\mathbf{x}}+\mathbf{B} \delta \dot{\mathbf{x}}+\mathbf{K} \delta \mathbf{x} & =\left[\begin{array}{c}
u_{\mathrm{pzt}}\left(V\left(t^{-} \mid t^{-}<t\right)\right)-u_{\mathrm{pzt}}\left(V_{\mathrm{pole}}(0)\right) \\
u_{\mathrm{g}}\left(\delta x_{\mathrm{p}}, \delta \dot{x}_{\mathrm{p}}, t\right)
\end{array}\right] \\
& =\left[\begin{array}{l}
\delta u_{\mathrm{c}}(t) \\
\delta u_{\mathrm{p}}(t)
\end{array}\right] .
\end{aligned}
$$

This formulation thus eliminates the effects of constant sources of strain from the model (such as residual strain in non-active thin films) and leaves for further identification models for piezoelectric forcing and ground interaction. Numeric values for $\mathbf{M}, \mathbf{B}$ and $\mathbf{K}$ are obtained from the conventional modal analysis with a low voltage (1 V) swept sine input, using the circle fit method [17]. The most prominent resonant peaks for the experimentally tested system occur at 623 and $6424 \mathrm{~Hz}$.

\subsection{PZT Model}

The forcing applied to the cantilever by the piezoelectric thin film as a function of voltage, equivalent to a net force applied to the cantilever beam relative to the reference force producing the neutral deflection after poling, i.e.

$$
\delta u_{\mathrm{c}}(t)=u_{\mathrm{pzt}}\left(V\left(t^{-} \mid t^{-}<t\right)\right)-u_{\mathrm{pzt}}\left(V_{\mathrm{pole}}(0)\right)
$$


from (5), is a very complex function of the voltage signal experienced by the PZT film up to the current time. This is especially true when both positive and negative electric fields are in use, as the polarization state of the film itself may vary from that achieved after poling. However, for strictly periodic input signals with a limited range of amplitudes used in the current work, it was found that the piezoelectric forcing could be approximated by comparatively simple and experimentally identifiable polynomial functions, such that

$$
\delta u_{\mathrm{c}}(t) \approx g_{\mathrm{c}}(V(t), \dot{V}(t)) .
$$

Here, $g_{\mathrm{c}}$ is an empirically fit hysteresis model for PZT actuation gain from static bending tests.

The function describing $g_{\mathrm{c}}$ is identified as a function of voltage by solving the equation

$$
K_{1,1} \delta x_{\mathrm{c}}=g_{\mathrm{c}}(V, \dot{V})
$$

for a number of voltage cycles and fitting polynomial curves to the hysteresis loops. Here, $K_{1,1}$ is the estimated stiffness of the cantilever from the structural model and $\delta x_{\mathrm{c}}$, the displacement of the center of the cantilever from its zero position, is being measured during static bending tests.

Figure 3(a) shows static displacements versus voltage for a cantilever beam stepped in increments from $0 \mathrm{~V}$ (after poling) back to $-8 \mathrm{~V}$, up to $8 \mathrm{~V}$, and back to $-8 \mathrm{~V}$. As it illustrates, the position of the proof mass is bound when the voltage reaches close to $8 \mathrm{~V}$ and it stays at the same position until the voltage falls back to $-2 \mathrm{~V}$. This is attributed to adhesion and this hypothesis is strengthened by the fact that the proof mass seems to be 'released' from the 'ground' surface when the voltage becomes negative. Figure $3(b)$ shows the second experimental data where the range of voltage is from -6 to $6 \mathrm{~V}$, which just avoids contact with ground for quasi-static motion and thus features no adhesion effect, as visible in the continuous changing in position of the proof mass during the voltage drop from 6 to $0 \mathrm{~V}$. While there is a substantial variation from cycle to cycle, as visible in figure $3(c)$, for the main purpose of contact modeling, an average empirical fit for $g_{c}$ was found as

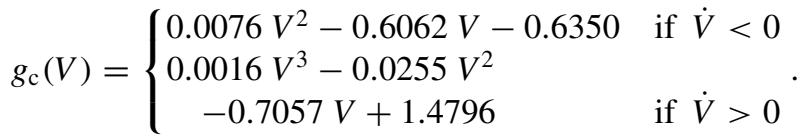

It should be noted that the full trajectory of the proof mass positions over a larger range of voltage would look like the plot on the bottom left corner of figure 3(c), which is a typical 'butterfly' feature of bipolar hysteresis of thin-film PZT actuators. For this study, only one side of the butterfly curve is in use, and the curve here is not symmetric along the $0 \mathrm{~V}$ line due to some inherent polarization of the PZT thin film during the fabrication process.

Because the error in the piezoelectric model can produce error in the parametric models for contact dynamics, at the conclusion of parameter identification a sensitivity analysis to alter the piezoelectric model is performed by varying the presumed zero position of the proof mass above the ground surface and repeating parameter identification as if that had been the true zero position of the structure (in effect shifting the piezoelectric gain to the extent of its experimental range).

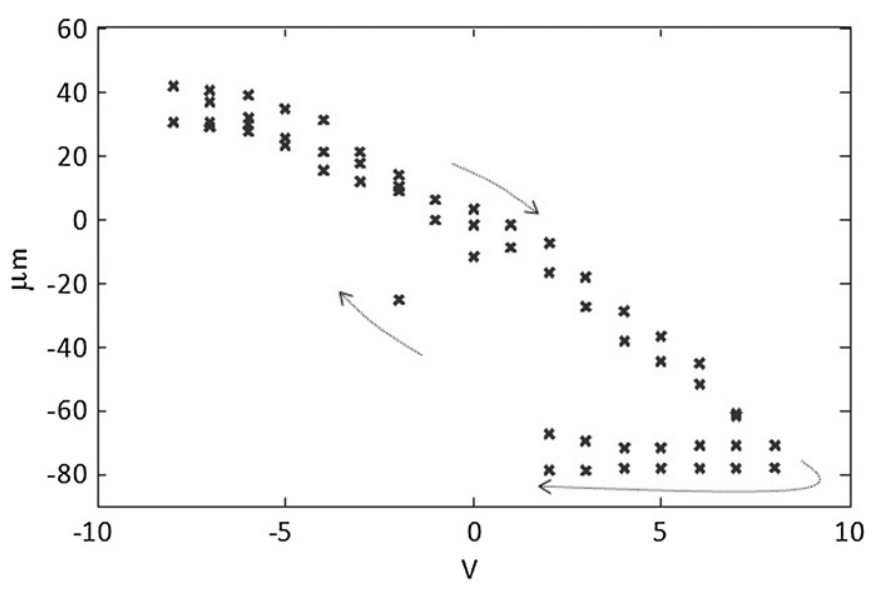

(a)

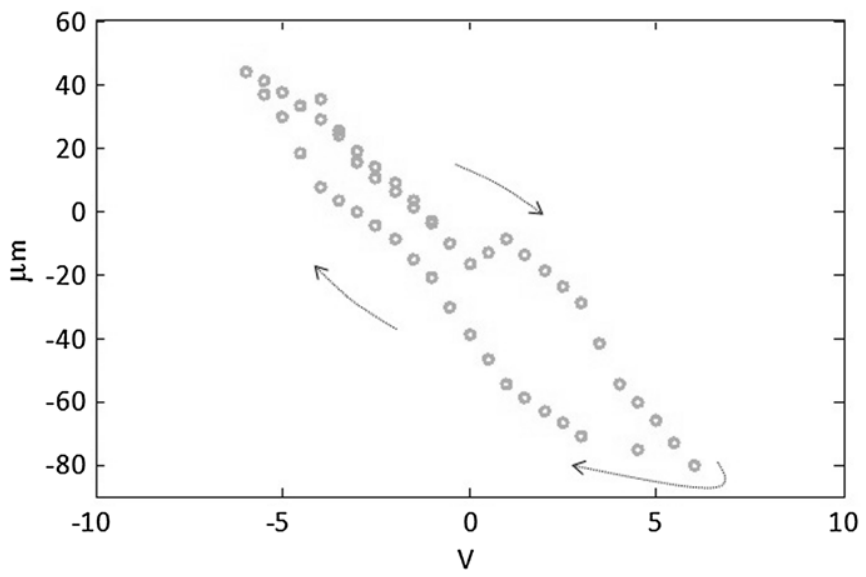

(b)

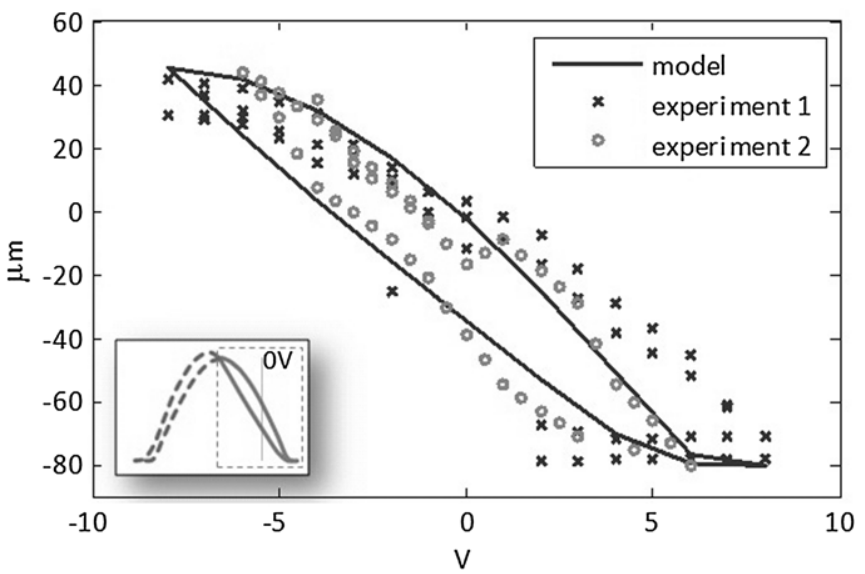

(c)

Figure 3. Hysteresis curves from cantilever testing here: (a) experiment 1 , voltage range $-8 \mathrm{~V}$ to $8 \mathrm{~V},(b)$ experiment 2 , voltage range $-6 \mathrm{~V}$ to $6 \mathrm{~V},(c)$ gain model and a fully interpolated hysteresis curve on the bottom left corner.

As will be discussed later, the contact model parameters prove relatively insensitive to changes in the piezoelectric model, although improved hysteresis modeling is a potential area of future work for modeling of the system as a whole, especially when a wider range of voltage amplitudes may be applied. 


\subsection{Impact modeling}

The goal of impact modeling is to identify a comparatively simple, parameterized model describing behavior when the proof mass at the tip of the cantilever impacts with the underlying 'ground'. Although this test structure used for model development can only interact with one surface, conceptually it should be possible to adjust the parameters describing the impact model to describe a variety of other surface interactions. The impact model developed here consists of lumped-parameter squeeze-film damping and adhesion models, together with a coefficient-of-restitution test for bouncing.

When the proof mass is not in contact with the ground, $\delta u_{p}(t)$ is estimated using a parameterized squeeze-film damping equation,

$$
\delta u_{\mathrm{p}}=C_{\mathrm{s}} \frac{\dot{x}_{\mathrm{p}}}{\left(x_{\mathrm{p}}+g_{\mathrm{p}}\right)^{3}}, \quad x_{\mathrm{p}}>-g_{\mathrm{p}},
$$

where $g_{\mathrm{p}}$ is the effective distance between the proof mass and ground at the zero position for the proof mass, and $C_{\mathrm{s}}$ is a lumped coefficient incorporating viscosity and other squeezefilm damping factors. $C_{\mathrm{s}}$ and $g_{\mathrm{p}}$ are tuned experimentally during the system-identification procedure in the following section. The value for $g_{\mathrm{p}}$ is not identical to the value for $d_{\mathrm{p}}$, which is the distance between the proof mass zero position and ground because the uneven shape of the underlying silicon results an effective non-zero gap when the proof mass is in contact, as shown schematically in figure 4.

If the proof mass comes into contact, the external groundinteraction force becomes a function of adhesion to ground, which is given in a general form:

$$
\delta u_{\mathrm{p}}=f_{\mathrm{a}}\left(t-t_{\mathrm{c}}\right), \quad x_{\mathrm{p}}=-d_{\mathrm{p}},
$$

where $t_{\mathrm{c}}$ is the time at which the current period of contact began, and $f_{\mathrm{a}}$ is an experimentally identified function for the adhesion force to be identified.

To determine whether the proof mass sticks or bounces at impact, a coefficient of restitution (CoR) model is used. First, at the moment of impact, the estimated bounce velocity is calculated from

$$
\dot{x}_{\mathrm{p}}^{+}=-\alpha \dot{x}_{\mathrm{p}}^{-}
$$

where $\dot{x}_{\mathrm{p}}^{-}$is the proof mass velocity just before impact, $\dot{x}_{\mathrm{p}}^{+}$is the hypothesized velocity after impact, and $\alpha$ is an experimentally determined CoR. Then, the pulling-off force associated with such a change in the velocity is calculated from the dynamics of the remainder of the system as

$$
f_{\text {pull }}=-K_{2,1} \delta x_{\mathrm{c}}-C_{2,1} \delta \dot{x}_{\mathrm{c}}+K_{2,2} \delta x_{\mathrm{p}}+C_{2,2} \delta \dot{x}_{\mathrm{p}} .
$$

If $f_{\text {pull }}$ is positive after the hypothesized bounce and is larger than $f_{\mathrm{a}}\left(t-t_{\mathrm{c}}\right)$, then the proof mass velocity is calculated from (12); otherwise, the proof mass is taken to be in sustained contact with ground, and the adhesion force from (11) is applied until $f_{\text {pull }}(t)$ at a future time exceeds $f_{\mathrm{a}}\left(t-t_{\mathrm{c}}\right)$.

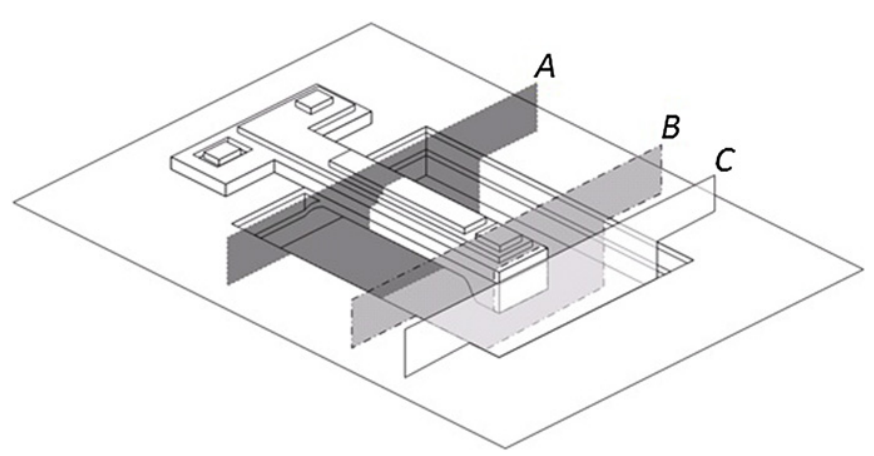

(a)

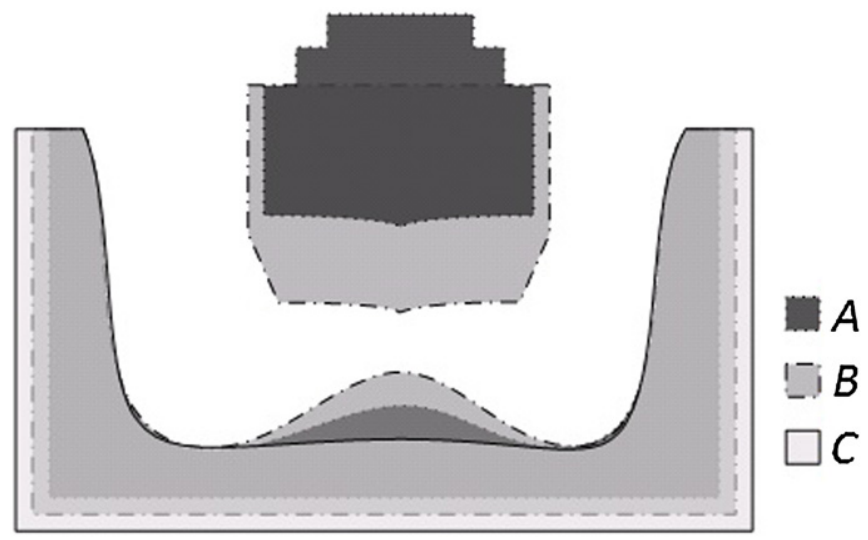

(b)

Figure 4. Cross-sectional diagram and expected ground surface: (a) schematic diagram of the test structure and (b) conceptual diagram of inferred cross-sections.

\section{Impact parameter identification}

Given that structural and piezoelectric models for the system from section 2 have been developed based on non-contact and static bending tests, three parameters and one function must be identified to finalize an impact model of the form proposed in (10)-(13). These are the squeeze-film coefficient and the effective gap between proof mass and ground, CoR and adhesion force function.

The CoR is most easily identified, by gradually increasing voltage to the system at a fixed oscillation frequency, in the present example $300 \mathrm{~Hz}$, until the proof mass just begins to impact the ground, without any sustained adhesion. A number of impact events are measured under the LDV, and the proof mass velocity is calculated before and after impact. The corresponding CoR is taken from the average value of many impacts, as shown in figure 5.

For adhesion and squeeze-film damping, a series of experiments was performed using ramped square waves. Defined in terms of frequency, voltage and a duty cycle variable, as shown in figure 6 , the ramped square waveform was found to be effective for producing comparatively gentle contact events with gradual changes in contact time for adhesion measurements. Furthermore, immediately after break-off, the ramp-up keeps the proof mass close to ground and accentuates squeeze-film damping effects.

To perform adhesion measurements, the pulling-off force from (13) acting on the proof mass is calculated using 


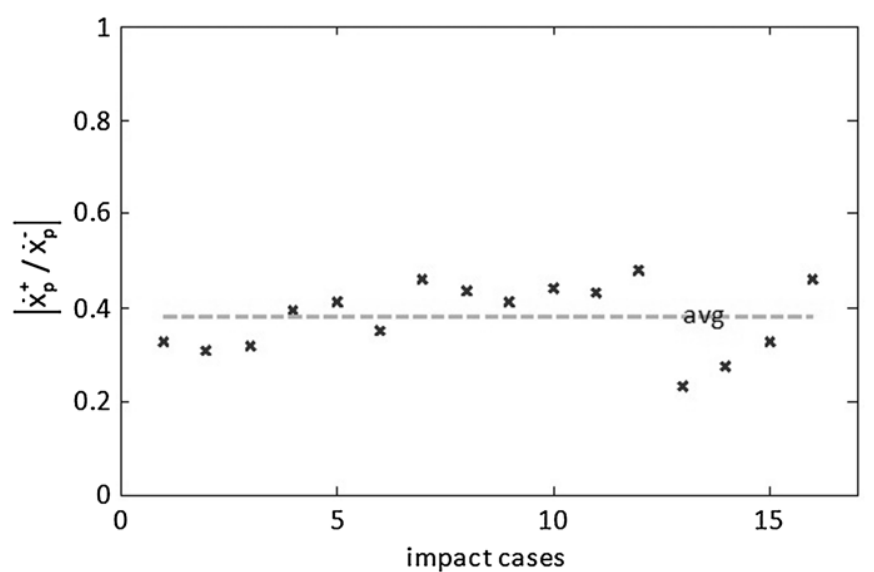

Figure 5. Ratio of proof mass velocity after impact to velocity before impact, for 16 sample bouncing events $(300 \mathrm{~Hz}$ square wave $/ 300 \mathrm{~Hz}$ sine wave).

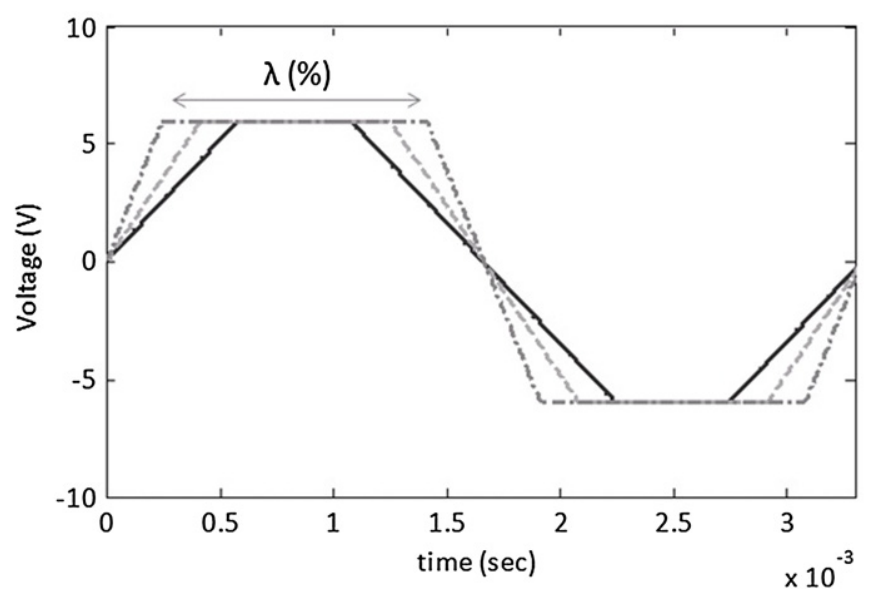

Figure 6. Sample ramped-square waveform used for adhesion and squeeze-film damping measurements.

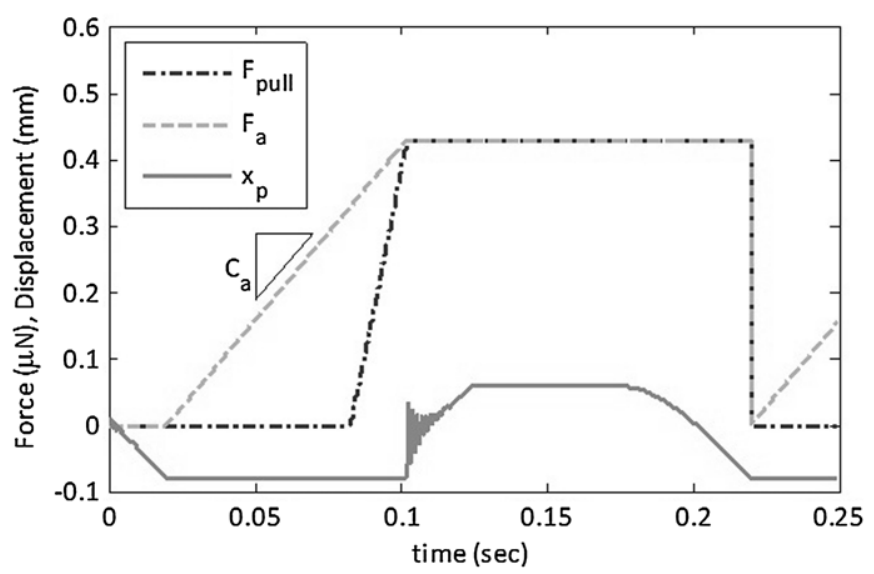

Figure 7. Sample proof mass position versus time with ramped-square wave, superimposed over inferred pull-off force.

the actuator model and observed cantilever and proof mass diaplacement over the duration of a single maintained contact period. Figure 7 shows a sample simulation study, showing calculated pull-off force and adhesion force versus the cantilever displacement.

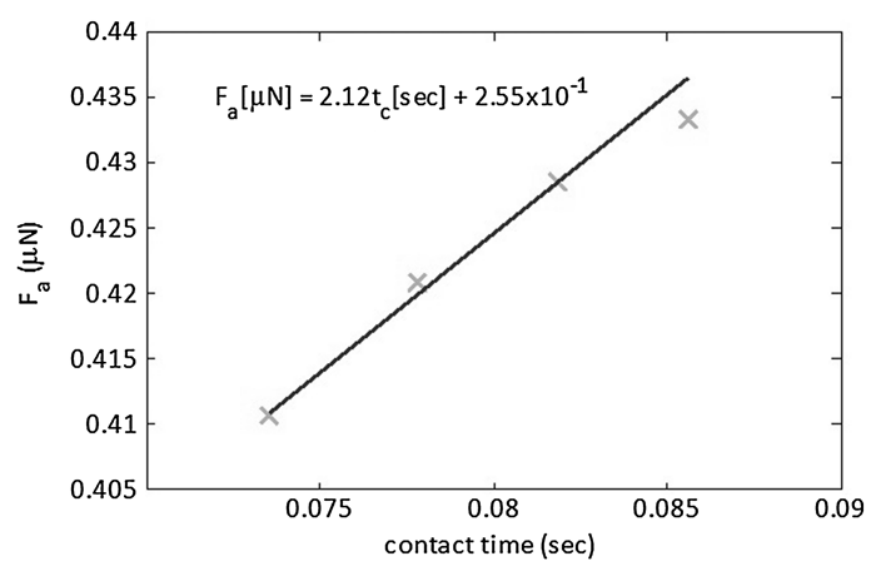

Figure 8. Inferred adhesion force versus contact duration for $5 \mathrm{~Hz}$ ramped-square waves.

Table 2. Coefficients for the impact model.

\begin{tabular}{|c|c|c|c|c|}
\hline \multirow[b]{2}{*}{ Coefficient } & \multicolumn{2}{|c|}{ Forcing scenarios $^{\mathrm{a}}$} & \multirow{2}{*}{$\begin{array}{l}\text { Related } \\
\text { phenomenon }\end{array}$} & \multirow[b]{2}{*}{ Unit } \\
\hline & Original & Altered & & \\
\hline$\alpha$ & 0.378 & 0.378 & CoR & - \\
\hline$C_{\mathrm{a}}$ & 1.700 & 2.120 & Adhesion & $\mu \mathrm{N} \mathrm{s}^{-1}$ \\
\hline$C_{\mathrm{a} 0}$ & $2.743 \times 10^{-1}$ & $2.550 \times 10^{-1}$ & Adhesion & $\mu \mathrm{N}$ \\
\hline$C_{\mathrm{s}}$ & $1.747 \times 10^{-15}$ & $1.747 \times 10^{-15}$ & $\begin{array}{l}\text { Squeeze-film } \\
\text { damping }\end{array}$ & $\mathrm{nN} \mathrm{m} \mathrm{m}^{2} \mathrm{~s}$ \\
\hline$g_{\mathrm{p}}$ & 58 & 58 & $\begin{array}{l}\text { Squeeze-film } \\
\text { damping }\end{array}$ & $\mu \mathrm{m}$ \\
\hline
\end{tabular}

${ }^{a}$ Two scenarios to check sensitivity by varying the idle position of the proof mass: $d_{\mathrm{p}}=70 \mu \mathrm{m}$ for the original model, and $d_{\mathrm{p}}=90 \mu \mathrm{m}$ for the altered model.

As shown in figure 7 , adhesion is taken to increase as contact time increases (with a linear trend proving consistent with experimental data), and the proof mass is released from the ground pulling-up force equal to the adhesion force. Figure 8 shows the adhesion force calculated from the pulloff force at break-off versus the contact duration for ramped square waves with varying $\lambda$ at $5 \mathrm{~Hz}$ and $8 \mathrm{~V}$ amplitude. While a relatively limited range of contact durations is obtained in this manner, a linear trend in the adhesion force with time is observed. This is consistent with some prior adhesion studies of microdevices, including [18-21], although the time periods of the present test are generally shorter (and adhesion force smaller) than in existing MEMS adhesion studies. The resulting adhesion model was identified using slope $C_{\mathrm{a}}$ and constant $C_{\mathrm{a} 0}$ as

$$
f_{\mathrm{a}}=C_{\mathrm{a}}\left(t-t_{\mathrm{c}}\right)+C_{\mathrm{a} 0} .
$$

Also visible in figure 7 is the oscillation of the proof mass after break-off, as it rises away from ground. This is a consequence of the sudden break-off against adhesion, and its peak amplitude and decay rate are used to fit the parameters of the simplified squeeze-film damping model in (10). As shown in figure 9, by adjusting the effective squeeze-film damping coefficient $C_{\mathrm{s}}$ and effective proof mass gap $g_{\mathrm{p}}$, such that the decay in oscillations are matched between the dynamic model and experimental results. Table 2 shows the coefficients obtained for the current test structure. Figure 10 shows an 


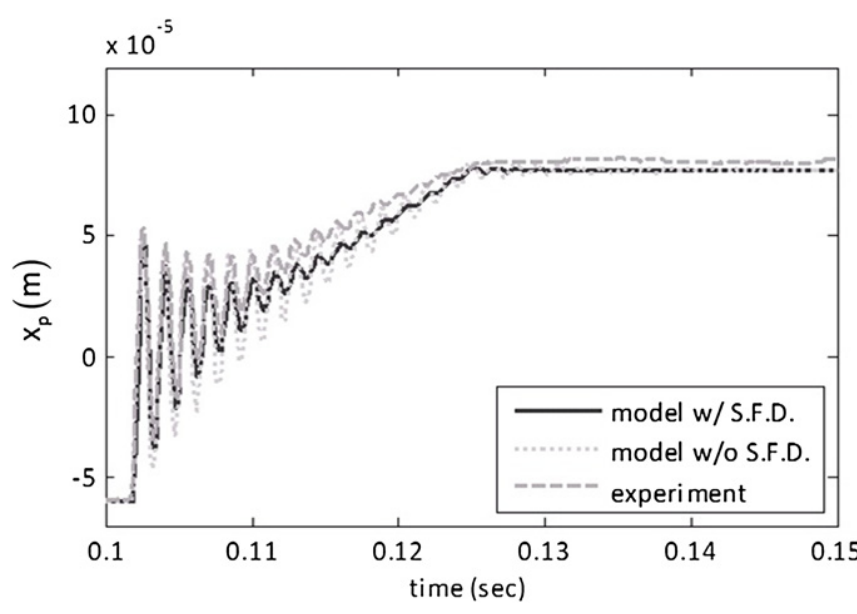

Figure 9. Comparison of decay in oscillations of displacement of the proof mass following break-off with and without squeeze-film damping incorporated in the dynamic contact model.

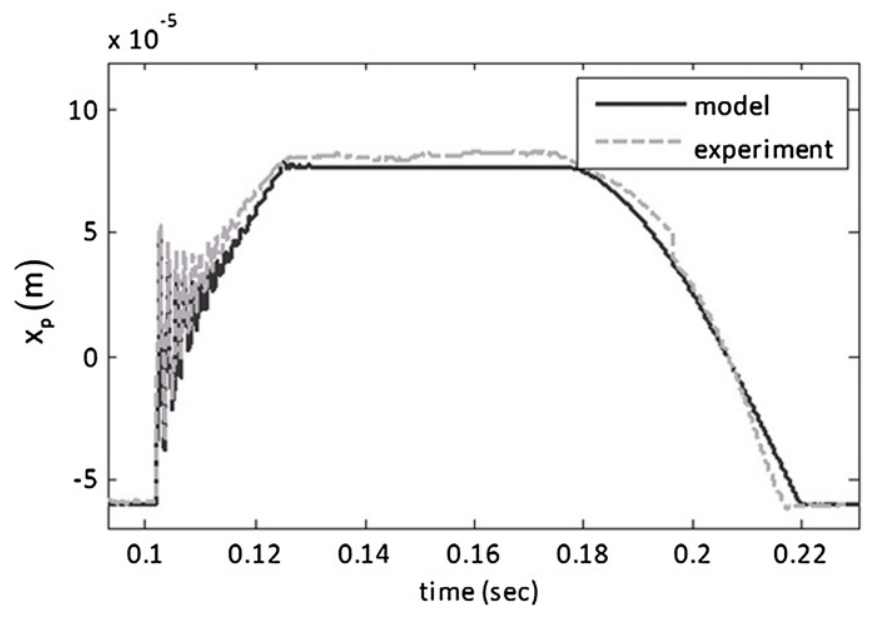

Figure 10. Sample response of experimental system and simulated system following model parameter identification to a ramped square wave $(5 \mathrm{~Hz}, 8 \mathrm{~V}, \lambda=25 \%)$.

example of the agreement between the dynamic model with identified coefficients and a sample ramped-square wave over a full cycle period.

To check the sensitivity of identified parameters to piezoelectric coefficient modeling, the above procedure was repeated assuming a piezoelectric gain function shifted by the equivalent of $20 \mu \mathrm{m}$ of zero position of the proof mass $d_{\mathrm{p}}$ shown in figure 11 . This offset was approximately equal to the maximum cycle-to-cycle deviation of static bending tests. Table 2 shows the comparison of contact dynamics model parameters under the two different piezoelectric forcing

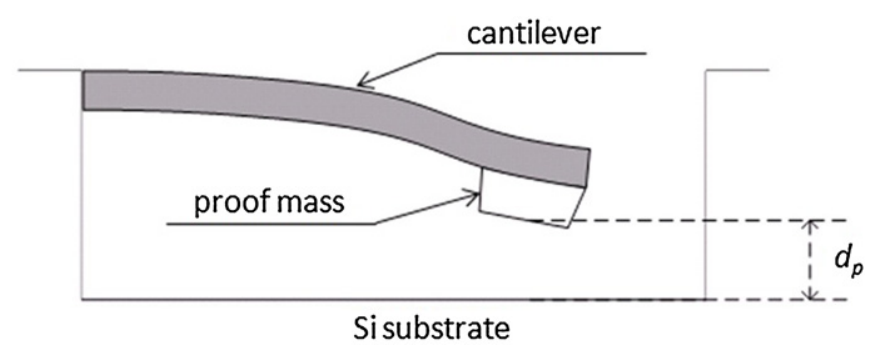

Figure 11. Idle position $d_{\mathrm{p}}$ : the distance between the proof mass and the substrate surface while the system is idle.

scenarios and this implies that the identified parameters are largely insensitive to the error of the estimated piezoelectric gain behavior.

\section{Model validation}

To verify that the contact model identified from static, simple bouncing and ramped-square wave experiments is effective for modeling a wider range of contact scenarios, several additional experiments were run with periodic input functions of varying form, frequency and amplitude. A summary of test cases of square or sine input and $6 \mathrm{~V}$ or $8 \mathrm{~V}$ driving voltage at $5,15,50$ and $100 \mathrm{~Hz}$ (16 cases total) is provided in table 3, with regard to the presence and number of bouncing events, the peak amplitude of oscillations after break-off and the contact duration. Results from the full 16 test cases are shown in the appendix. The ranges of tested frequencies and waveforms were chosen considering the practical voltage inputs for micro-robots, which are typically low-power frequency square waves, with sine waves as a possible alternate. Voltages were limited to 6 and $8 \mathrm{~V}$ to avoid non-contact cases at lower voltages and higher voltage cases where piezoelectric driving force overpowered small-scale phenomena. Although some centimeter-scale micro-robots may utilize resonant frequencies, it was assumed that simple waveforms with lower frequencies would be still helpful to validate the contact model due to comparatively large time duration of contact interaction with the ground.

The simplified, lumped parameter model identified to describe system contact dynamics captures the majority of major events seen in the validation tests. The presence or the absence of bouncing events was predicted correctly in all cases, although some predictions give a mismatch for the number of bounces in the cases at lower voltage inputs. About $60 \%$ of all test cases showed the identical number of bounces. Contact duration predictions are generally good, with error not greater than $30 \%$ in more than half of the test cases. The contact duration is least reliable for the cases of lower voltage

Table 3. Summary of model validation results.

\begin{tabular}{lllllll}
\hline $\begin{array}{l}\text { Existence of } \\
\text { bounce } \\
\text { prediction } \\
\text { correctly }\end{array}$ & $\begin{array}{l}\text { Number of } \\
\text { bounces } \\
\text { correctly }\end{array}$ & $\begin{array}{l}\text { Average error in } \\
\text { number of } \\
\text { bounces }\end{array}$ & $\begin{array}{l}\text { Average error in } \\
\text { peak oscillation } \\
\text { amplitude }\end{array}$ & $\begin{array}{l}\text { Cases with error } \\
\text { below 30\% }\end{array}$ & $\begin{array}{l}\text { Average error in } \\
\text { contact duration }\end{array}$ & $\begin{array}{l}\text { Cases with error } \\
\text { below 30\% }\end{array}$ \\
\hline $100 \%$ & $75 \%$ & 0.28 & $-9 \%$ & $75 \%$ & $20 \%$ & $62.5 \%$ \\
\hline
\end{tabular}




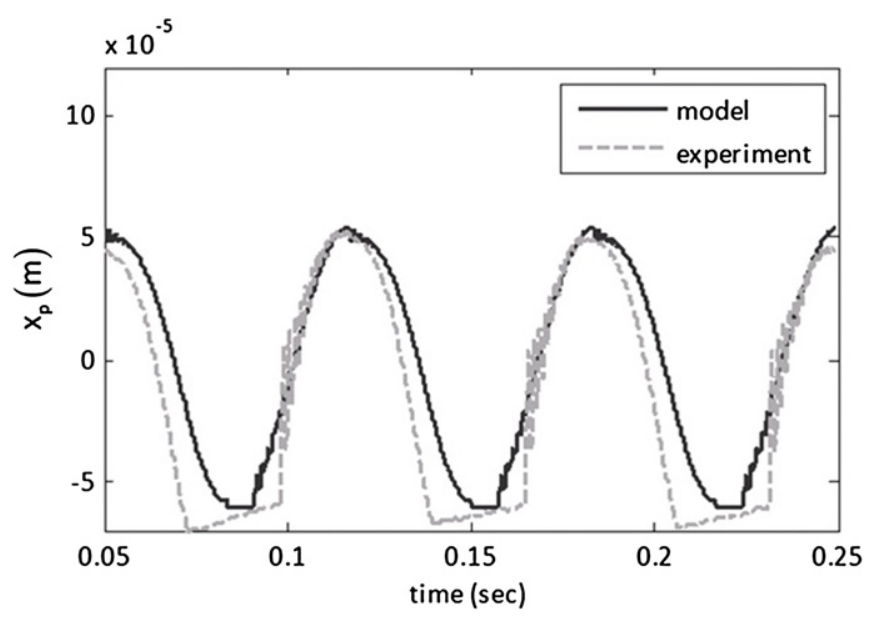

(a)

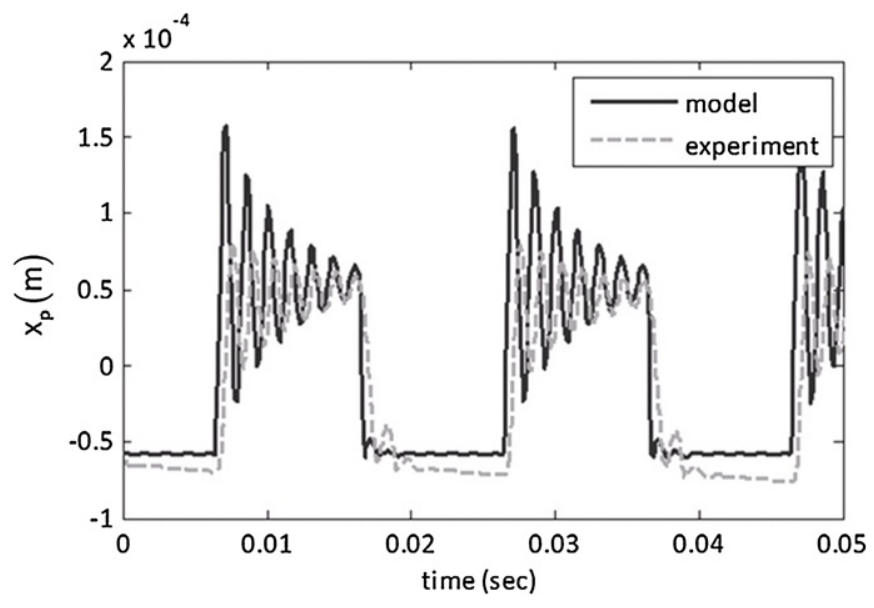

(b)

Figure 12. Sampling of validation test signal results, worst cases: (a) sine wave $6 \mathrm{~V} 15 \mathrm{~Hz}$ and (b) square wave $6 \mathrm{~V} 50 \mathrm{~Hz}$.

input, with substantial errors seen in a few test cases. The peak oscillation error was lower than $30 \%$ in nearly all cases, with greater errors observed only for some square-wave tests. Errors are attributed primarily to unmodeled minor vibration modes, particularly when excited by step inputs, and also to the complicated hysteretic behavior of piezoelectric actuation. An error may also arise from some small contribution of electrostatic attraction, primarily at higher frequencies; in the experimental setup, the electrostatic force is minimized as a result of grounding the bottom PZT electrode, but if any electrostatic force exists, only its static effects are accounted for through the influence of electrostatic attraction on the calculated piezoelectric gain function. Nonetheless, even the worse test cases give a fair agreement with overall periodic time response, as shown in figures $12(a)$ and $(b)$, despite the numerically quantified errors. Overall, $50 \%$ of all test cases showed less than $30 \%$ error in both peak oscillation error and contact duration predictions, and again, the presence or the absence of bouncing was predicted correctly in all cases.

Other representative responses are also shown in figure 13. Figure 13(a) shows the very close agreement between simulation and experimental results at low frequencies, using identified model parameters. At higher frequencies, such as the $50 \mathrm{~Hz}$ results in figures $13(b)$ and $(c)$, greater differences occur, with unmodeled minor vibration modes especially evident in the response to an $8 \mathrm{~V}$ sinusoidal input in figure 13(c). This difference, however, does not severely increase even in higher driving frequencies as seen in figure $13(d)$ : the general response amplitude is quite close even when higher mode oscillation amplitudes are off. Generally speaking, the match between simulation and real system behavior over the entire duration of periodic validation trajectories appears unusually good for a micro-scale dynamic contact model.

\section{Discussion}

The goal of this work was to develop a relatively simple model for contact dynamics of a microdevice, along with a procedure to quantify parameters in the model. Simply, in this context, a primarily lumped-parameter model with a limited numbers of parameters has been indicated, rather than potentially more accurate distributed or finite-element models. The major benefit of the completed model after parameter identification is that it provides good prediction in the simulation of a variety of experimentally measured transient and periodic phenomena.

The ability to replicate a variety of dynamic responses is unusual for a model of contact dynamics of a MEMS device with relatively large areas subject to surface interactions. Also important is that the model can be obtained when information about device geometries and surface conditions is limited. This model approach is able to do this, though, because it uses a subset of experimental system inputs and measured responses to quantify coefficients in a generic system model, rather than attempting to make predictions from specific system geometries and material properties. This means that the approach used here has a limited utility for predicting behavior at the design stage of a given device and is not at this time able to provide insight into the effects of material choices. On the other hand, once systematic identification is made, response of the system to alternative inputs, as provided by feedforward or feedback control, or perturbations to the contact model, made through adjustments to model parameters, can be estimated.

Regarding the specific model contents proposed in this paper, the main difference from most previous work with large contact areas is the use of piezoelectric actuation, such that electrostatic forces are less prominent than in other applications, and squeeze-film damping and adhesion forces tend to be more important. Meanwhile, while piezoelectric actuation is common in the more precisely defined contact interactions of atomic force microscopy and similar techniques, contact geometries studied here are much different. The forms for squeeze-film damping and adhesion forces have been chosen based on common forms used in references treating devices in dimensions similar to the microcantilever test structure tested in this paper, but reduced when possible so that each force is formulated with a simple few coefficients. In terms of more general applications, the form for squeeze-film damping is not unusual. Meanwhile, the exact functional form (linear fit) of the adhesion force model may be dependent on the material combination used, though at least some region of linearly increasing adhesion force with contact 


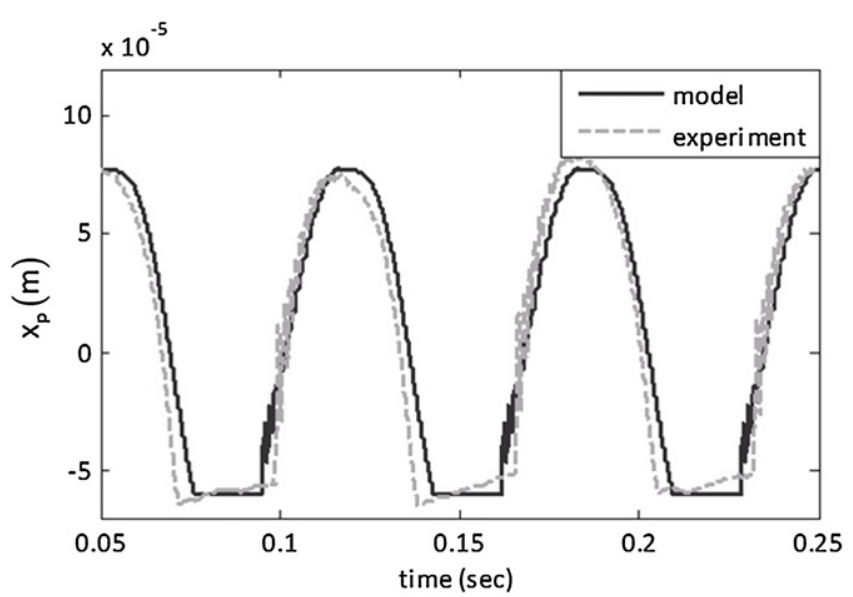

(a)

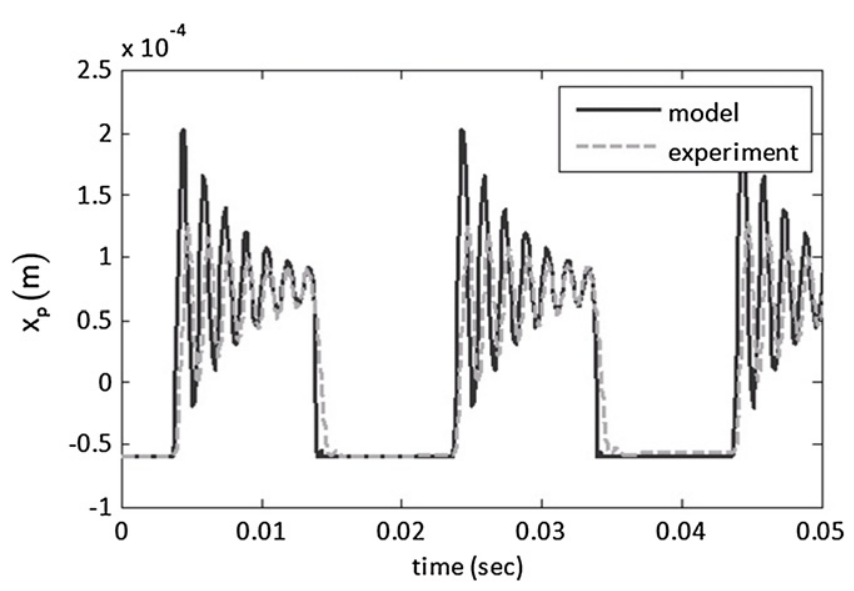

(c)

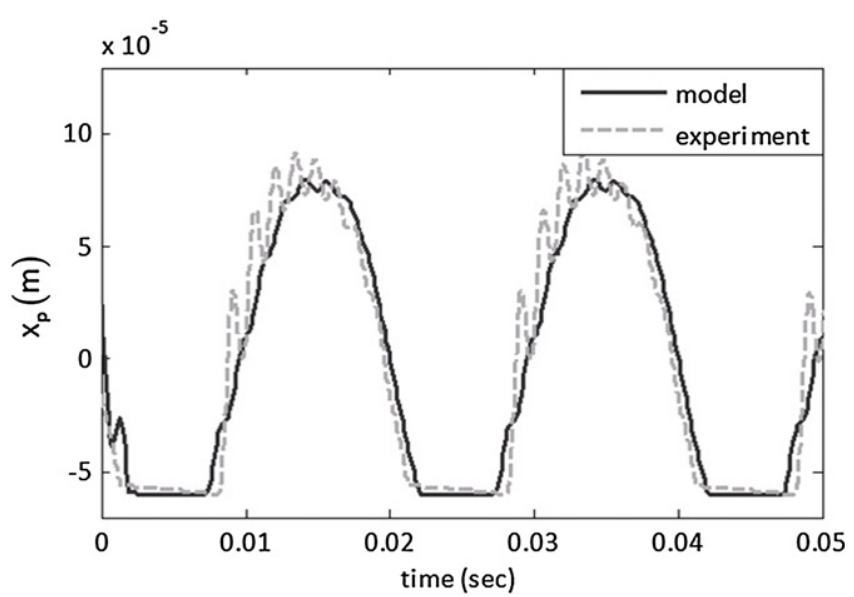

(b)

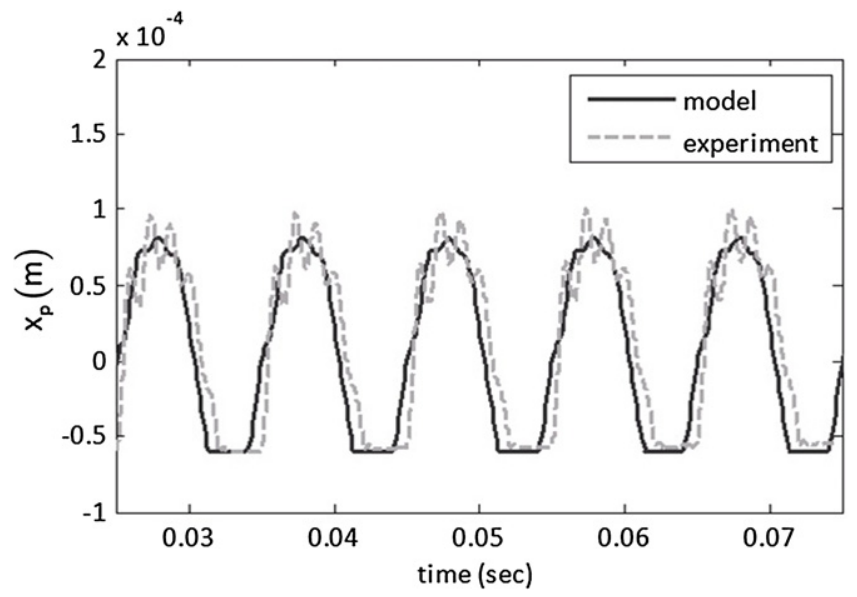

$(d)$

Figure 13. Sampling of validation test signal results, best cases: (a) sine wave $8 \mathrm{~V} 15 \mathrm{~Hz},(b)$ sine wave $8 \mathrm{~V} 50 \mathrm{~Hz},(c)$ square wave $8 \mathrm{~V}$ $50 \mathrm{~Hz}$ and $(d)$ sine wave $8 \mathrm{~V} 100 \mathrm{~Hz}$.

time is often observed in other research, and the procedure for generating the pull-off force is generally applicable. Likewise, the hysteresis model for the PZT thin film used here is entirely case specific, and much more sophisticated hysteresis models could replace it in other applications. Additionally, since electrostatic force is obscured in this hysteresis model, its contribution to the dynamics of the structure is ignored. This may contribute to error in responses under high driving frequencies. Hence, further development of the hysteresis model including the dynamic behavior of electrostatic force might reduce error. Finally, coefficient-of-restitution models for bouncing behavior have been applied to microsystems previously, but prove effective again in this work.

Similarly, the system-identification approach proposed has some nice generally applicable attributes, and a few aspects that are not necessarily applicably in all cases. The provided sequence generally separates individual phenomena, although certain phenomena are not entirely isolated, such as hysteresis measurements and electrostatic forces. Likewise, if applied to other devices may not be able to reduce close range effects by shifting the neutral position of other devices in the same manner that a piezoelectric cantilever can be polarized in opposite directions. One aspect of the procedure that can be useful to estimate the forces like adhesive force and squeeze- film damping force is the ramped-square input signal with various slopes, which has been designed and applied to the system.

\section{Conclusion}

Using a cantilever-type thin-film piezoelectric actuator, this work introduces a procedure for model identification of contact dynamics of a piezoelectric microsystem without full knowledge of contact surface geometry. The model uses simple lumped-parameter components that whose parameters can be identified with relatively simple experimentation. Certain piezoelectric linearities plus squeeze-film damping and adhesion effects and a coefficient-of-restitution characterizing the surfaces in question are incorporated into the model. Experimental validation indicates that the model overall provides a fair approximation of transient and periodic behavior over various driving frequencies and waveforms in time series responses. Effects that are captured well include the amplitude and phase shifts in response to sinusoidal inputs, the presence or the absence of bouncing at contact and the attenuation rates of transient oscillations. The peak amplitude of oscillation, duration of contact and high mode 
Table A.1. Sampling of 16 test cases.

\begin{tabular}{|c|c|c|c|c|c|c|c|}
\hline Frequency $(\mathrm{Hz})$ & Voltage (V) & Signal & $\begin{array}{l}\text { Number of } \\
\text { bounces }\end{array}$ & $\begin{array}{l}\text { Peak oscillation } \\
\text { amplitude }(\mu \mathrm{m})\end{array}$ & Error & $\begin{array}{l}\text { Contact } \\
\text { duration (ms) }\end{array}$ & Error \\
\hline \multirow[t]{2}{*}{5} & \multirow[t]{2}{*}{6} & \multirow[t]{2}{*}{ Square } & Sim: 2 & Sim: 211 & \multirow[t]{2}{*}{$-13 \%$} & Sim: 97 & \multirow[t]{2}{*}{$1 \%$} \\
\hline & & & Exp: 3 & Exp: 186 & & Exp: 98 & \\
\hline \multirow[t]{2}{*}{5} & \multirow[t]{2}{*}{6} & \multirow[t]{2}{*}{ Sine } & Sim: 1 & Sim: 114 & \multirow[t]{2}{*}{$9 \%$} & Sim: 31 & \multirow[t]{2}{*}{$58 \%$} \\
\hline & & & Exp: 1 & Exp: 125 & & Exp: 75 & \\
\hline \multirow[t]{2}{*}{5} & \multirow[t]{2}{*}{8} & \multirow[t]{2}{*}{ Square } & Sim: 1 & Sim: 264 & \multirow[t]{2}{*}{$-25 \%$} & Sim: 99 & \multirow[t]{2}{*}{$-2 \%$} \\
\hline & & & Exp: 1 & Exp: 210 & & Exp: 97 & \\
\hline \multirow[t]{2}{*}{5} & \multirow[t]{2}{*}{8} & \multirow[t]{2}{*}{ Sine } & Sim: 0 & Sim: 136 & \multirow[t]{2}{*}{$-7 \%$} & Sim: 72 & \multirow[t]{2}{*}{$12 \%$} \\
\hline & & & Exp: 0 & Exp: 146 & & Exp: 82 & \\
\hline \multirow[t]{2}{*}{15} & \multirow[t]{2}{*}{6} & \multirow[t]{2}{*}{ Square } & Sim: 0 & Sim: 216 & \multirow[t]{2}{*}{$8 \%$} & Sim: 0 & \multirow[t]{2}{*}{$0 \%$} \\
\hline & & & Exp: 0 & Exp: 236 & & Exp: 0 & \\
\hline \multirow[t]{2}{*}{15} & \multirow[t]{2}{*}{6} & \multirow[t]{2}{*}{ Sine } & Sim: 0 & Sim: 114 & \multirow[t]{2}{*}{$8 \%$} & Sim: 7 & $70 \%$ \\
\hline & & & Exp: 0 & Exp: 124 & & Exp: 24 & \\
\hline 15 & 8 & Square & Sim: 0 & Sim: 264 & $-24 \%$ & Sim: 33 & $-3 \%$ \\
\hline & & & Exp: 0 & Exp: 212 & & Exp: 32 & \\
\hline 15 & 8 & Sine & Sim: 0 & Sim: 138 & $-7 \%$ & Sim: 19 & $26 \%$ \\
\hline & & & Exp: 0 & Exp: 148 & & Exp: 26 & \\
\hline 50 & 6 & Square & Sim: 5 & Sim: 212 & $-32 \%$ & Sim: 2.7 & $47 \%$ \\
\hline & & & Exp: 3 & Exp: 160 & & Exp: 5.1 & \\
\hline 50 & 6 & Sine & Sim: 1 & Sim: 114 & $21 \%$ & Sim: 1.8 & $67 \%$ \\
\hline & & & Exp: 2 & Exp: 94 & & Exp: 5.4 & \\
\hline 50 & 8 & Square & Sim: 1 & Sim: 252 & $-42 \%$ & Sim: 9.2 & $-15 \%$ \\
\hline & & & Exp: 1 & Exp: 184 & & Exp: 8 & \\
\hline 50 & 8 & Sine & Sim: 1 & Sim: 140 & $8 \%$ & Sim: 5.1 & $21 \%$ \\
\hline & & & Exp: 1 & Exp: 152 & & Exp: 6.5 & \\
\hline 100 & 6 & Square & Sim: 0 & Sim: 205 & $-34 \%$ & Sim: 0 & $0 \%$ \\
\hline & & & Exp: 0 & Exp: 152 & & Exp: 0 & \\
\hline 100 & 6 & Sine & Sim: 1 & Sim: 117 & $15 \%$ & Sim: 0.4 & $78 \%$ \\
\hline & & & Exp: 1 & Exp: 137 & & Exp: 1.8 & \\
\hline 100 & 8 & Square & Sim: 1 & Sim: 264 & $-36 \%$ & Sim: 4.2 & $-35 \%$ \\
\hline & & & Exp: $1 \sim 2$ & Exp: 194 & & Exp: 3.1 & \\
\hline 100 & 8 & Sine & Sim: 1 & Sim: 142 & $9 \%$ & Sim: 2.6 & $-13 \%$ \\
\hline & & & Exp: 1 & Exp: 156 & & Exp: 2.3 & \\
\hline
\end{tabular}

oscillation in the air at a driving frequency faster than $50 \mathrm{~Hz}$ are less well modeled, although in most cases the agreement of experimental and simulated responses is quite good.

The results of this modeling effort will next be applied to more practical device applications. The primary application area is micro-robotics, where even though it is difficult to predict coefficients before hand over many terrains, for design of gait inputs a designer can look at the response to system inputs over a range of coefficients and pick input sequences producing a robust response. In other circumstances, it may be possible to perform partial or complete identification, while initiating locomotion and using the resulting information to provide trajectories with desirable behavior.

\section{Acknowledgments}

The authors thank the US Army Research Laboratory and Choong-Ho Rhee at the University of Michigan for fabrication of the cantilever test structure, and the National Science Foundation award CMMI 0954422 for support for this work.

\section{Appendix. Sampling of all test cases}

A full set of measurements from 16 validation test cases is shown in table A.1.

\section{References}

[1] Lee S I, Howell S W, Raman A and Reifenberger R 2002 Nonlinear dynamics of microcantilevers in tapping mode atomic force microscopy: a comparison between theory and experiment Phys. Rev. B 66115409

[2] Hagleitner C, Bonaccio T, Rothuizen $\mathrm{H}$ and Eleftheriou E 2007 Modeling, design, and verification for the analog front-end of a MEMS-based parallel scanning-probe storage device IEEE J. Solid-State Circuits 8 1779-89

[3] Guo Z J, McGruer N E and Adams G G 2007 Modeling, simulation and measurement of the dynamic performance of an Ohmic contact, electrostatically actuated RF MEMS switch J. Micromech. Microeng. 17 1899-909

[4] Field R V and Epp D S 2006 Development and calibration of a stochastic dynamics model for the design of a MEMS inertial switch Sensors Actuators A 134 109-18

[5] Teodurescu M, Theodossiades S and Rahnejat H 2008 Impact dynamics of rough and surface protected MEMS gears Tribol. Int. 42 197-205

[6] Wang T and Asokanthan S F 2011 Bouncing dynamics in MEMS based switching structures Proc. Eur. Nonlinear Dynamics Conf. (Rome, Italy)

[7] Decuzzi P, Demelio G P, Pascazio G and Zaza V 2006 Bouncing dynamics of resistive microswitch with an adhesive tip J. Appl. Phys. 100024313

[8] Do C, Cychowski M, Lishchynska M, Hill M and Delaney K 2010 Integrated modeling of nonlinear dynamics and 
contact mechanics of electrostatically actuated RF-MEMS switches Annu. Conf. IEEE Industrial Electronics Society (Glendale, AZ) pp 2293-8

[9] Park Y H, Miyazaki Y and Park K C 2003 High fidelity MEMS switch model Nanotechnology 1 416-9

[10] LaRose R P and Murphy K D 2009 Impact dynamics of MEMS switches Nonlinear Dyn. 60 327-39

[11] McCarthy B, Adams G G, McGruer N E and Potter D 2002 A dynamic model, including contact bounce, of an electrostatically actuated microswitch $J$. Microelectromech. Syst. 11 276-83

[12] Granaldi A and Decuzzi P 2006 The dynamic response of resistive microswitches: switching time and bouncing J. Micromech. Microeng. 161108

[13] Zhang W, Baskaran R and Turner K 2003 Tuning the dynamic behavior of parametric resonance in a micromechanical oscillator Appl. Phys. Lett. 82130

[14] Czaplewski D A, Dyck C W, Sumali H, Maddad J E, Kuppers J D, Reines I, Cowan W D and Tigges C P 2006 A soft-landing waveform for actuation of a single-pole single-throw RF MEMS switch J. Microelectromech. Syst. 15 1586-94

[15] Oldham K, Rhee C-H, Ryou J H, Polcawich R and Pulskamp J 2009 Lateral thin-film piezoelectric actuators for bio-inspired micro-robotic locomotion Proc. ASME Microand Nano-Systems Symp. (San Diego, CA)

[16] Qiu Z, Pulskamp J, Lin X, Rhee C-H, Wang T, Polcawich R and Oldham K 2010 Large displacement vertical translational actuator based on piezoelectric thin films J. Micromech. Microeng. 20075016

[17] Oldham K, Pulskamp J, Polcawich R and Dubey M 2008 Thin-film PZT lateral actuators with extended stroke J. Microelectromech. Syst. 17 890-9

[18] Maia N M M and Silva J M M 2001 Modal analysis identification techniques Phil. Trans. R. Soc. A $35929-40$

[19] Bhushan B, Liu H and Hsu S M 2004 Adhesion and friction studies of silicon and hydrophobic and low friction films and investigation of scale effects J. Tribol. 126 583-90

[20] Tambe N S and Bhushan B 2004 Scale dependence of micro/nano-friction and adhesion of MEMS/NEMS materials, coatings and lubricants Nanotechnology $151561-70$

[21] Luengo G, Pan J, Heuberger M and Israelachvili J N 1998 Temperature and time effect on the adhesion dynamics of poly(butyl methacrylate) (PBMA) surfaces Langmuir 14 3873-81

[22] Persson B, Albohr O, Tartaglino U, Volokitin A I and Tosatti E 2005 On the nature of surface roughness with application to contact mechanics, sealing, rubber friction and adhesion J. Phys.: Condens. Matter 17 R1-62

[23] Taya M, Almajid A A, Dunn M and Takahashi H 2003 Design of bimorph piezo-composite actuators with functionally graded microstructure Sensors Actuators A 107 248-60

[24] Wagg D J 2006 A note on coefficient of restitution models including the effects of impact induced vibration J. Sound Vib. 300 1071-8 